\title{
Giant Prepyloric Ulcer Haemorrhage: Patient Characteristics, Treatment, and Outcome in 2003-2012
}

\author{
Margot Peetsalu, Ülle Kirsimägi, and Ants Peetsalu \\ Surgery Clinic of Tartu University Hospital, L. Puusepa 8, 51014 Tartu, Estonia \\ Correspondence should be addressed to Margot Peetsalu; margot.peetsalu@kliinikum.ee
}

Received 30 September 2013; Revised 7 March 2014; Accepted 21 March 2014; Published 10 April 2014

Academic Editor: Tomasz Brzozowski

Copyright (c) 2014 Margot Peetsalu et al. This is an open access article distributed under the Creative Commons Attribution License, which permits unrestricted use, distribution, and reproduction in any medium, provided the original work is properly cited.

\begin{abstract}
The aim of the study was to characterize patients with giant prepyloric ulcer haemorrhage, their treatment, and outcome. The data for 929 peptic ulcer haemorrhage (PUH) patients were divided into three groups according to ulcer location: group I: 165 prepyloric ulcer patients, group II: 353 gastric ulcer patients, and group III: 411 duodenal ulcer patients. Among these groups giant ulcer (ulcer diameter $\geq 2 \mathrm{~cm}$ ) haemorrhage cases were selected for the study and the data for group I were compared with the data for groups II and III. The study shows that the proportion of giant ulcers in all prepyloric ulcers was significantly 2.2 times lower compared with the proportion of giant ulcers in all gastric ulcers and 1.5 times lower than the proportion of giant ulcers in all duodenal ulcers. The share of surgical therapy for haemorrhages from giant prepyloric ulcers did not differ from the share of surgical therapy in the case of giant gastric ulcers but was 3.5 times lower than in the case of giant duodenal ulcers. Generally, treatment for PUH from giant prepyloric ulcers was successful not involving inhospital mortality.
\end{abstract}

\section{Introduction}

Haemorrhage is the most frequent complication of peptic ulcer disease. Despite advances in endoscopic and pharmacological treatment in peptic ulcer haemorrhage (PUH) the mortality associated with this condition remains around 5\% according to recent multicentre studies $[1,2]$.

The problem connected with the diagnostics and treatment of PUH is the increasing proportion of elderly $(\geq 65)$ patients frequently suffering from concomitant diseases. Concomitant diseases and aging involve use of drugs favouring ulcer development and haemorrhage. In addition, the prognosis of PUH is negatively affected also by other factors: early haemorrhage recurrence and ulcer size $\geq 2 \mathrm{~cm}$ both in isolation and in combination [3-5].

It is well known that endoscopic haemostasis is the cornerstone method in the treatment of PUH. The most common cause of failure of endoscopic haemostasis is the difficulty to access the source of haemorrhage. This is due to extensive scarring and deformation in presence of giant peptic ulcer but usually not in presence of standard size peptic ulcer. Endoscopic treatment fails more often in the case of haemorrhage from giant peptic ulcers, particularly duodenal posterior wall ulcers penetrating into the pancreas, compared with haemorrhage from giant gastric ulcers [6, 7]. Gastric ulcers differ in their location, secretory status, and cause [8]. There is only scanty information about prepyloric peptic ulcer haemorrhage as it is pathogenetically grouped under duodenal ulcer haemorrhage [9] or has not been classified in more detail [10-13].

Yet the incidence of prepyloric ulcer haemorrhage is significantly lower than the incidence of gastric or duodenal ulcer haemorrhage [14]. However, there are no data about PUH from giant prepyloric peptic ulcers.

The aim of the present study was to characterize patients with giant prepyloric peptic ulcer haemorrhage, their treatment, and outcome during 10 years (2003-2012).

\section{Material and Methods}

We analysed the data for all 953 acute peptic ulcer haemorrhage patients, aged $\geq 15$ years (1053 hospitalised PUH cases) 
and treated for PUH at the Surgery Clinic, Tartu University Hospital, during 10 years (2003-2012).

The PUH cases were identified from the patient registry representing discharge diagnosis according to codes in the International Classification of Diseases (ICD-10). Starting with "K25" and "K26" coding for gastric and duodenal ulcer, respectively, all PUH patients' medical records, endoscopy protocols, and operative or autopsy records were evaluated.

Haemorrhage was defined as hematemesis and/or melena, a drop in the haemoglobin/haematocrit value, or clinical evidence of blood loss, confirmed at endoscopy, operation, or autopsy. The PUH was defined as circumscribed mucosal break of at least $5 \mathrm{~mm}$ in diameter with a well-defined ulcer crater and with interpretation of the intensity of haemorrhage according to the Forrest scale.

The PUH was classified in accordance with ulcer location: haemorrhage from gastric ulcer (gastric corpus ulcer), prepyloric ulcer (within 0.5 to $3 \mathrm{~cm}$ proximal of the pyloric ring), pyloric ulcer, or duodenal ulcer as described earlier [14]. The PUH from giant ulcer was defined as haemorrhage from an ulcer with a size of $\geq 2 \mathrm{~cm}$ as described earlier [15]. Ulcer size was estimated at endoscopy, using biopsy forceps, or on the basis of operative or autopsy data. The intensity of haemorrhage was assessed according to the wellknown Forrest scale: class I: active bleeding (spurting or oozing vessel); class II: ulcer with signs of recent haemorrhage (thrombosed visible vessel or adherent clot); and class III: ulcer without signs of recent haemorrhage (flat pigmented spots or clean ulcer base). High risk stigmata haemorrhage was classified as Forrest class I and class II as described earlier [16].

Emergency endoscopy for alimentary tract haemorrhage is the responsibility of four experienced general surgeons of the Surgery Clinic and it has been available roundthe-clock at the clinic since 1988. To stop haemorrhage, injection methods were commonly used, depending on the decision of the attending physician. Combination therapy with adrenalin and ethanol was used in half of the cases and monotherapy with ethanol or adrenalin was equally used in the remaining cases. A repeat endoscopic procedure was applied in clinically suspected PUH recurrence after its endoscopic confirmation. In single cases, haemostatic clips or electrocoagulation was used. Transarterial embolisation was employed in two cases. As a rule, before and after the endoscopic procedure, histamine 2 receptor antagonists and, since 2008, proton pump inhibitors were administered.

In our study of acute PUH cases, endoscopic biopsy from ulcer for histopathological examination and from the antrum mucosa for $H$. pylori examination was not routinely performed. Patients whose endoscopy with gastrobiopsy study (in malignancy suspected cases) or operative treatment or autopsy showed malignancy were excluded from the study.

Any information about drug use potentially associated with poor outcome of haemorrhage (nonsteroidal antiinflammatory drugs (NSAIDs), low and high dose aspirin, synthetic steroids, and vitamin $\mathrm{K}$ antagonists) was obtained from medical records.

The indication for surgery in the case of PUH was mainly ineffective medical and endoscopic treatment. In single cases, so-called early surgical treatment was used for high-risk haemorrhage recurrence patients (before recurrent PUH) after primary endoscopic treatment as recommended [17].

The aim of the current study was a more thorough analysis of PUH from giant prepyloric ulcer (patient characteristics, treatment, and outcome) compared with PUH from giant gastric ulcer and giant duodenal ulcer. For this purpose, haemorrhage cases from pyloric ulcers (24) were excluded from all 953 PUH cases. The remaining 929 PUH patients were divided into three groups with giant ulcer selection: group I: 24 giant prepyloric ulcer patients (of all 165 prepyloric ulcer patients), group II: 115 giant gastric ulcer patients (of all 353 gastric ulcer patients); and group III: 92 giant duodenal ulcer patients (of all 411 duodenal ulcer patients).

\section{Statistics}

Statistical analysis was conducted using the software Statistica 12.0. Continuous variables are presented as mean $\pm \mathrm{SD}$ (standard deviation of the mean), and categorical variables are presented as percentages (\%). The $\chi^{2}$ and Fisher's exact tests were used to assess differences between categorical data, and the $t$-test was used to assess differences between continuous data. A value of $P \leq 0.05$ was considered to indicate statistical significance, all $P$ values were two-sided.

\section{Results}

In group I the proportion of giant ulcers in all prepyloric ulcers was significantly lower compared with group II, $14.6 \%$ $(24 / 165)$ versus $32.6 \%(115 / 353)(P=0.0001)$, and with group III, $14.6 \%$ versus $22.4 \%(92 / 411)(P=0.02)$.

Table 1 shows that mean patient age in group I was 68.9 years, which is not significantly different from mean patient age in groups II and III. The proportion of patients older than 65 years was higher in group I than in group II, $66.7 \%$ versus $43.5 \%(P=0.02)$, but did not differ from the corresponding indicator for group III.

The data for group I regarding previous hospitalisations for PUH (29.2\%), use of haemorrhage favouring drugs (75.0\%), or endoscopically high-risk stigmata cases (70.8\%) were not significantly higher compared with the corresponding indicators for groups II and III (Table 1).

Table 2 presents data about treatment and outcome. Endoscopic treatment in the case of Forrest I-II was used in $88.2 \%$ of the cases in group I versus $80.9 \%$ in group II $(P=0.49)$ and versus $82.9 \%$ in group III $(P=0.73)$. Surgical treatment was employed in $12.5 \%$ of the cases in group I versus $12.2 \%$ in group II and versus $43.5 \%$ in group III ( $P=$ 0.002 ). There was no postoperative inhospital mortality in group I; however, in group II and group III it accounted for $7.1 \%$ and $7.5 \%$ of the cases, respectively. According to the data of total mortality for both medically + endoscopically and surgically treated patients, there were no postoperative death cases in group I, while $3.5 \%$ of the patients in group II and $5.4 \%$ of the patients in group III died (Table 2). 
TABLE 1: Patients' characteristics for giant peptic ulcer haemorrhage: group I (study group): giant prepyloric ulcer haemorrhage; group II: giant gastric ulcer haemorrhage; group III: giant duodenal ulcer haemorrhage.

\begin{tabular}{lccc}
\hline Characteristics & $\begin{array}{c}\text { Group I } \\
\text { Patients (\%) }\end{array}$ & $\begin{array}{c}\text { Group II } \\
\text { Patients (\%) }\end{array}$ & 115 \\
Patients (\%)
\end{tabular}

Drug use: potentially associated with poor outcome of haemorrhage; PUH: peptic ulcer haemorrhage; Forrest I-II = I: spurting or oozing bleeding; II: visible vessel or adherent clot; ${ }^{*}$ significant difference between group I and group II.

TABLE 2: Treatment methods and outcome for giant peptic ulcer haemorrhage: group I: giant prepyloric ulcer haemorrhage; group II: giant gastric ulcer haemorrhage; group III: giant duodenal ulcer haemorrhage.

\begin{tabular}{|c|c|c|c|}
\hline Patients $(n)$ & $\begin{array}{c}\text { Group I } n(\%) \\
24 \\
\end{array}$ & $\begin{array}{l}\text { Group II } n(\%) \\
115 \\
\end{array}$ & $\begin{array}{l}\text { Group III } n(\%) \\
92 \\
\end{array}$ \\
\hline \multicolumn{4}{|l|}{ Treatment methods } \\
\hline Endoscopic treatment in Forrest I-II cases & $15 / 17(88.2)$ & $76 / 94(80.9)$ & $58 / 70(82.9)$ \\
\hline Repeat endoscopic treatment & $3 / 15(20.0)$ & 9/76 (11.8) & $17 / 58(29.3)$ \\
\hline Surgical treatment & $3(12.5)^{+}$ & $14(12.2)$ & $40(43.5)$ \\
\hline \multicolumn{4}{|l|}{ Outcome } \\
\hline Postoperative mortality & 0 & $1 / 14(7.1)$ & $3 / 40(7.5)$ \\
\hline Mortality (total) & 0 & $4(3.5)$ & $5(5.4)$ \\
\hline
\end{tabular}

Forrest I-II = I: spurting bleeding or oozing bleeding; II: visible vessel or adherent clot; ${ }^{+}$significant difference between group I and group III.

\section{Discussion}

According to the data of the present study, the proportion of giant ulcers in all ulcers in PUH varies depending on ulcer localisation. The proportion of giant ulcers in all prepyloric ulcers is $14.6 \%$, which is significantly 2.2 times lower than the proportion of giant ulcers in all gastric ulcers, $32.6 \%$, and significantly 1.5 times lower than the proportion of giant ulcers in all duodenal ulcers, $22.4 \%$. It shows that the proportion of giant prepyloric ulcers is slightly closer to that of giant duodenal ulcers than to that of giant gastric ulcers. It has been shown earlier that prepyloric ulcers are more similar to duodenal than to gastric ulcers owing mainly to pathogenetically higher gastric acid secretion as well as to other indicators $[8,18]$. Development of gastric ulcer is primarily associated with the weakened defence capability of the gastric mucosa and normal or lower gastric acid secretion. At the same time, it is known that peptic ulcer disease is heterogeneous regarding its etiology and pathogenesis. According to general knowledge, important factors in the etiology of peptic ulcer are $H$. pylori infection and NSAID use [19]. Still, the role of these factors may vary between different ulcer locations [20]. This was also demonstrated in our earlier prospective study of $\mathrm{PUH}$ : presence of $H$. pylori depending on ulcer location was $100 \%, 81 \%$, and $93 \%$ and NSAID use was $42.9 \%, 25.8 \%$, and $13.5 \%$ in the case of prepyloric, gastric, and duodenal haemorrhage cases, respectively [14].

Up to now, data accounting for differences in pathophysiology that lead a certain patient to develop these giant duodenal ulcer variants have been scanty [21]. Different explanations involve genetic predisposition, dietary or environmental factors, microbial influence, variations in immunological response, or any combination of these factors [22, 23], besides $H$. pylori infection and NSAID use. Patients with giant gastric ulcers are reported to be more unwell and older than patients with nongiant ulcers. Malnutrition 
is known to affect ulcer healing as it reduces the integrity of the gastric mucosa. Patients with giant gastric ulcers have significantly lower serum protein level than patients with small ulcers [24].

Although the indicators that in isolation or in combination significantly worsened the prognosis of PUH from giant prepyloric ulcers in our study were higher (mean patient age 69 years, rate of previous hospitalisations due to PUH $29 \%$, and rate of haemorrhage favouring drug use $75 \%$ ), this difference was not significant compared with PUH from giant gastric or duodenal ulcers. However, the proportion of older patients ( $>65$ years) in giant prepyloric ulcer cases was significantly higher compared with the proportion of older patients in giant gastric ulcer cases (66.7\% versus $43.5 \%$ ) but not significantly higher compared with the same indicator for giant duodenal ulcer cases (60.9\%). This indicates differences in giant ulcer cases depending on ulcer location in the stomach. Older age has also been reported as an independent risk factor for poor outcome [3].

It is known that ulcer location within proximity to large arteries, like gastric ulcers in the left gastric artery area and duodenal posterior wall ulcers in the gastroduodenal artery area, is a more predictive factor for recurrent haemorrhage and poor outcome $[25,26]$ in comparison with other areas. Giant gastric ulcers at the lesser stomach curvature can erode the left gastric artery and duodenal posterior wall ulcers can erode the gastroduodenal artery. The size of these arteries or their first generation branches exceeds the limits of endoscopic haemostasis methods [27]. Often pseudoaneurysms form overeroded arteries, which can be considerably larger than the artery proper [28].

Considering the above data, our expected endoscopic finding in giant gastric and duodenal ulcer cases was the more frequent occurrence of high risk stigmata haemorrhage in comparison with giant prepyloric haemorrhage cases. Although the proportion of such haemorrhages was indeed somewhat higher in the giant gastric and duodenal ulcer cases, $81.7 \%$ and $76.1 \%$, respectively, it was still not significantly higher than the corresponding indicator for giant prepyloric ulcer cases, $70.8 \%$. Neither was there significant difference in the use of endoscopic treatment and repeat endoscopic treatment between haemorrhages from the above locations.

Our study shows that PUH from giant ulcers can be managed in most cases without surgery using medical treatment in combination with endoscopic treatment. Surgical treatment to stop haemorrhage was needed in $12.5 \%$ of the giant prepyloric ulcer cases and in $12.2 \%$ of the giant gastric ulcer cases but significantly 3.5 times less than in the giant duodenal ulcer cases (43.5\%).

These data confirm that the success of medical treatment in combination with endoscopic treatment in the case of PUH from giant prepyloric or gastric ulcers was significantly higher than in the case of PUH from giant duodenal ulcers. This result can be expected, as in the aspect of stopping bleeding successful endoscopic haemostasis is of utmost importance. However, this is far more difficult to achieve in cases of giant duodenal ulcers compared with giant prepyloric or gastric ulcers, as has been reported also earlier [6].
Our study shows that estimated postoperative mortality; that is, death rate during hospital treatment (inhospital mortality) in the cases of PUH from giant gastric and duodenal ulcers was $7.1 \%$ and $7.5 \%$, respectively, without deaths in the cases of PUH from giant prepyloric ulcers. Also total mortality during medical + endoscopic and surgical therapy involved no deaths in giant prepyloric ulcer cases but was $3.5 \%$ in giant gastric ulcer cases and $5.4 \%$ in giant duodenal ulcer cases. This can be considered an important finding in the treatment outcome of PUH from giant prepyloric ulcers in comparison with giant gastric or giant duodenal ulcers.

\section{Conclusion}

Our study shows that in PUH cases there are some differences between haemorrhages from giant prepyloric ulcers compared with haemorrhages from giant gastric or duodenal ulcers: the proportion of giant prepyloric ulcers in all prepyloric ulcers was two times lower compared with the proportion of giant gastric ulcers in all gastric ulcers and 1.5 times lower from the proportion of giant duodenal ulcers in all duodenal ulcers; the share of surgical treatment in the cases of PUH from giant prepyloric ulcers was more than threefold lower than the share in the cases of PUH from giant duodenal ulcers. Treatment outcome in PUH from giant prepyloric ulcers was more successful, that is, without inhospital mortality, compared with treatment outcome in PUH both from giant gastric and duodenal ulcers. It shows that the treatment of PUH from giant gastric and duodenal ulcers is more complicated compared with the treatment of haemorrhages from giant prepyloric ulcers.

\section{Ethical Approval}

The study was approved by the Ethics Committee of the University of Tartu, Estonia, no. 188T-3 (14.12.2009).

\section{Conflict of Interests}

The authors declare that there is no conflict of interests regarding the publication of this paper.

\section{References}

[1] A. Barkun, C. A. Fallone, N. Chiba et al., "A Canadian clinical practice algorithm for the management of patients with nonvariceal upper gastrointestinal bleeding," Canadian Journal of Gastroenterology, vol. 18, no. 10, pp. 605-609, 2004.

[2] R. Marmo, M. Koch, L. Cipolletta et al., "Predictive factors of mortality from nonvariceal upper gastrointestinal hemorrhage: a multicenter study," American Journal of Gastroenterology, vol. 103, no. 7, pp. 1639-1647, 2008.

[3] S. Christensen, A. Riis, M. Nørgaard, H. T. Sørensen, and R. W. Thomsen, "Short-term mortality after perforated or bleeding peptic ulcer among elderly patients: a population-based cohort study," BMC Geriatrics, vol. 7, article 8, 2007.

[4] C.-Y. Chang, M.-S. Wu, C.-T. Lee et al., "Prospective survey for the etiology and outcome of peptic ulcer bleeding: a community 
based study in Southern Taiwan," Journal of the Formosan Medical Association, vol. 110, no. 4, pp. 223-229, 2011.

[5] P. García-Iglesias, A. Villoria, D. Suarez et al., "Meta-analysis: predictors of rebleeding after endoscopic treatment for bleeding peptic ulcer," Alimentary Pharmacology and Therapeutics, vol. 34, no. 8, pp. 888-900, 2011.

[6] K. C. Thomopoulos, J. A. Mitropoulos, E. C. Katsakoulis et al., "Factors associated with failure of endoscopic injection haemostasis in bleeding peptic ulcers," Scandinavian Journal of Gastroenterology, vol. 36, no. 6, pp. 664-668, 2001.

[7] J. Lipschitz, G. R. Gecelter, M. G. Veller, J. R. Botha, and J. A. Myburgh, "Successes and failures of endoscopic injection haemostasis for bleeding peptic ulcers. A prospective clinical study," South African Journal of Surgery, vol. 31, no. 4, pp. 132136, 1993.

[8] J. C. Padussis and T. N. Pappas, "Management of bleeding peptic ulcer disease," in Gastrointestinal Bleeding: Practical Approach To Diagnosis and Management, A. D. Pryor, T. N. Pappas, and M. S. Branch, Eds., pp. 39-64, 2010.

[9] C. Mel Wilcox, "Features associated with painless peptic ulcer bleeding," American Journal of Gastroenterology, vol. 92, no. 8, pp. 1289-1292, 1997.

[10] P. W. Y. Chiu, E. K. W. Ng, S. K. H. Wong et al., "Surgical salvage of bleeding peptic ulcers after failed therapeutic endoscopy," Digestive Surgery, vol. 26, no. 3, pp. 243-248, 2009.

[11] R. Czymek, A. Großmann, U. Roblick et al., "Surgical management of acute upper gastrointestinal bleeding: still a major challenge," Hepato-Gastroenterology, vol. 59, no. 115, pp. 768773, 2012.

[12] J. P. Hreinsson, E. Kalaitzakis, S. Gudmundsson, and E. S. Bjornsson, "Upper gastrointestinal bleeding: incidence, etiology and outcomes in a population-based setting," Scand $J$ Gastroenterol, vol. 48, no. 4, pp. 439-447, 2013.

[13] S. Ishikawa, T. Inaba, M. Wato, S. Takashima, T. Mizushige, K. Izumikawa et al., "Exposed blood vessels of more than $2 \mathrm{~mm}$ in diameter are a risk factor for rebleeding after endoscopic clipping hemostasis for hemorrhagic gastroduodenal ulcer," Digestive Endoscopy, vol. 25, no. 1, pp. 13-19, 2013.

[14] J. Soplepmann, A. Peetsalu, M. Peetsalu, A. Tein, and M. Juhola, "Peptic ulcer haemorrhage in Tartu county, Estonia: epidemiology and mortality risk factors," Scandinavian Journal of Gastroenterology, vol. 32, no. 12, pp. 1195-1200, 1997.

[15] D. M. Simeone, A. Hassan, and J. M. Scheiman, "Giant peptic ulcer: a surgical or medical disease?” Surgery, vol. 126, no. 3, pp. 474-478, 1999.

[16] A. Lanas, M. Polo-Tomas, S. Garcia, L. A. Garcia-Rodriguez, J. L. Calleja, X. Calvet et al., "Outcomes of peptic ulcer bleeding, with high- or low-risk stigmata, following treatment with proton pump inhibitors in routine clinical practice," Gut, vol. 61, 3, p. A380, 2012.

[17] M. H. Schoenberg, "Surgical therapy for peptic ulcer and nonvariceal bleeding," Langenbeck's Archives of Surgery, vol. 386, no. 2, pp. 98-103, 2001.

[18] M.-S. Wu, H.-P. Wang, J.-T. Wang, T.-H. Wang, and J.-T. Lin, "Serum pepsinogen I and pepsinogen II, and the ratio of pepsinogen II pepsinagen II in peptic ulcer diseases: with special emphasis an the influence of the location of the ulcer crater," Journal of Gastroenterology and Hepatology, vol. 10, no. 4, pp. 401-404, 1995.

[19] A. Shiotani and D. Y. Graham, "Pathogenesis and therapy of gastric and duodenal ulcer disease," Medical Clinics of North America, vol. 86, no. 6, pp. 1447-1466, 2002.
[20] M.-H. Chen, M.-S. Wu, W.-C. Lee, H.-P. Wang, and J.-T. Lin, "A multiple logistic regression analysis of risk factors in different subtypes of gastric ulcer," Hepato-Gastroenterology, vol. 49, no. 44, pp. 589-592, 2002.

[21] E. B. Newton, M. R. Versland, and T. E. Sepe, "Giant duodenal ulcers," World Journal of Gastroenterology, vol. 14, no. 32, pp. 4995-4999, 2008.

[22] P. E. T. Arkkila, A. Kokkola, K. Seppälä, and P. Sipponen, "Size of the peptic ulcer in Helicobacter pylori-positive patients: association with the clinical and histological characteristics," Scandinavian Journal of Gastroenterology, vol. 42, no. 6, pp. 695701, 2007.

[23] A. Karpouza, E. Samouilidou, S. Karagiannis et al., "Patients with duodenal ulcer have lower levels of serum cholesterol compared to other dyspeptic patients independently of Helicobacter pylori status," Scandinavian Journal of Gastroenterology, vol. 43, no. 8, pp. 922-928, 2008.

[24] A. Mizuki, M. Tatemichi, H. Nishiya et al., "Mucosal concentration of basic fibroblast growth factor in the healing process in human giant gastric ulcers," Journal of Gastroenterology and Hepatology, vol. 19, no. 5, pp. 528-534, 2004.

[25] C. P. Swain, P. R. Salmon, and T. C. Northfield, "Does ulcer position influence presentation or prognosis of upper gastrointestinal bleeding?" Gut, vol. 27, p. A632, 1986.

[26] E. Brullet, R. Campo, X. Calvet, D. Coroleu, E. Rivero, and J. Simó Deu, "Factors related to the failure of endoscopic injection therapy for bleeding gastric ulcer," Gut, vol. 39, no. 2, pp. 155158, 1996.

[27] B. J. Elmunzer, S. D. Young, J. M. Inadomi, P. Schoenfeld, and L. Laine, "Systematic review of the predictors of recurrent hemorrhage after endoscopic hemostatic therapy for bleeding peptic ulcers," American Journal of Gastroenterology, vol. 103, no. 10, pp. 2625-2632, 2008.

[28] J. Lau and J. J. Y. Sung, "From endoscopic hemostasis to bleeding peptic ulcers: strategies to prevent and treat recurrent bleeding," Gastroenterology, vol. 138, no. 4, pp. 1252.el-1254.e1, 2010. 


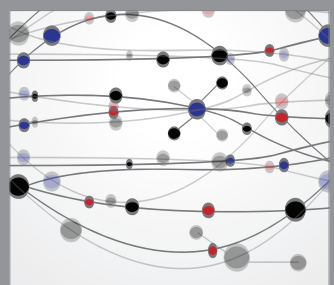

The Scientific World Journal
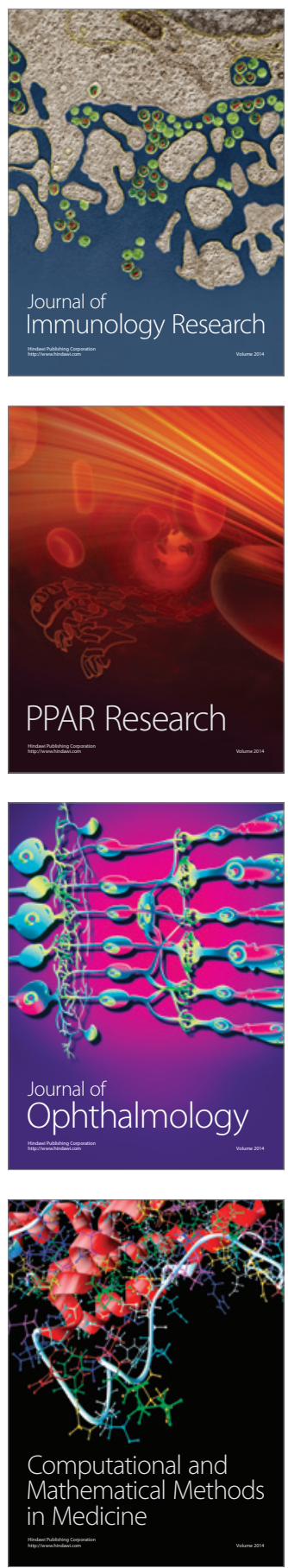

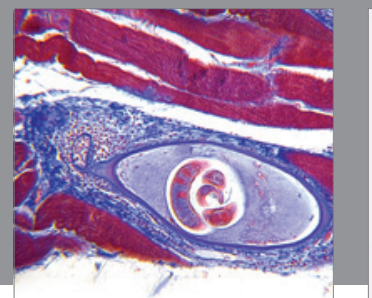

Gastroenterology

Research and Practice
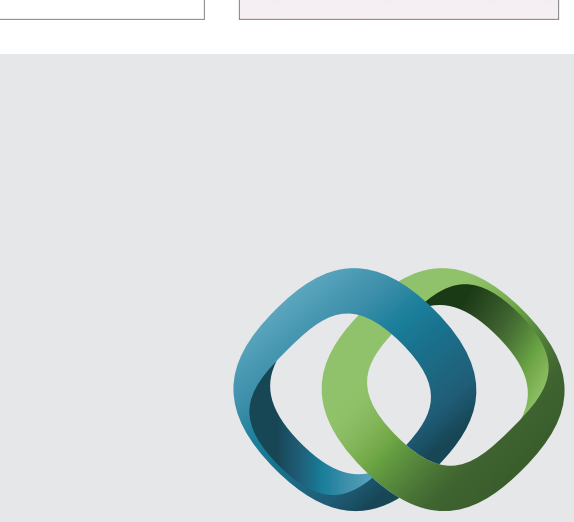

\section{Hindawi}

Submit your manuscripts at

http://www.hindawi.com
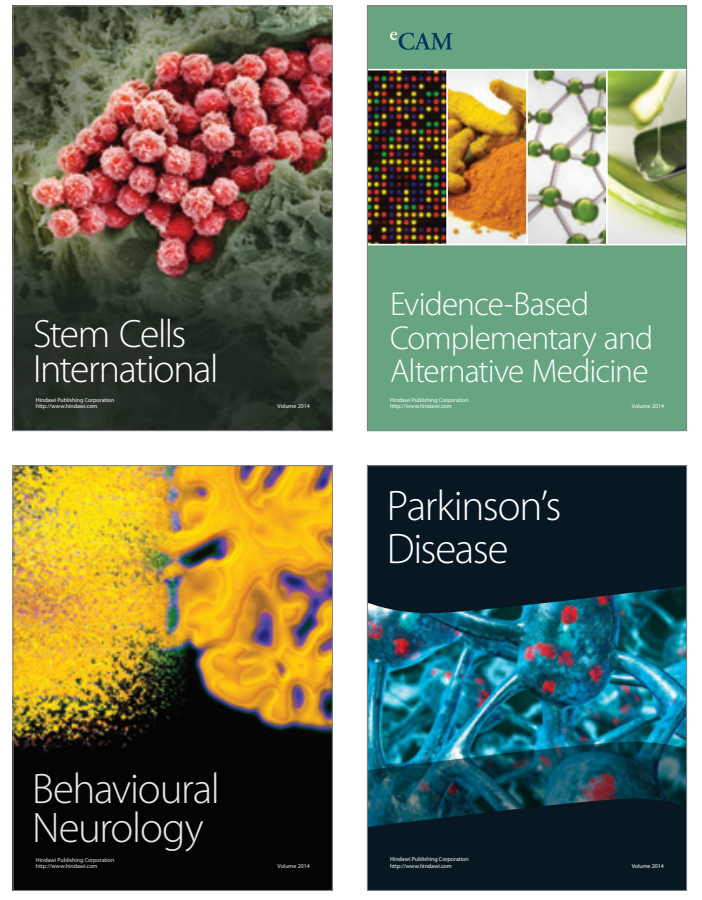
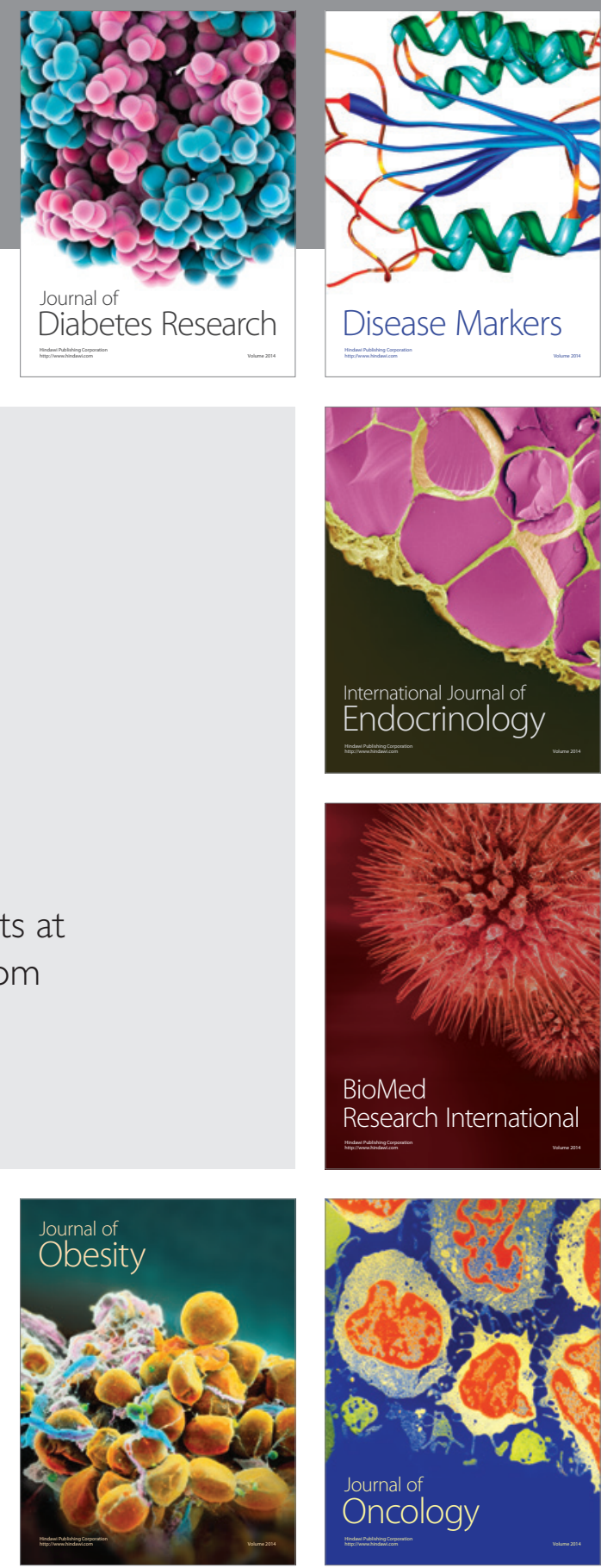

Disease Markers
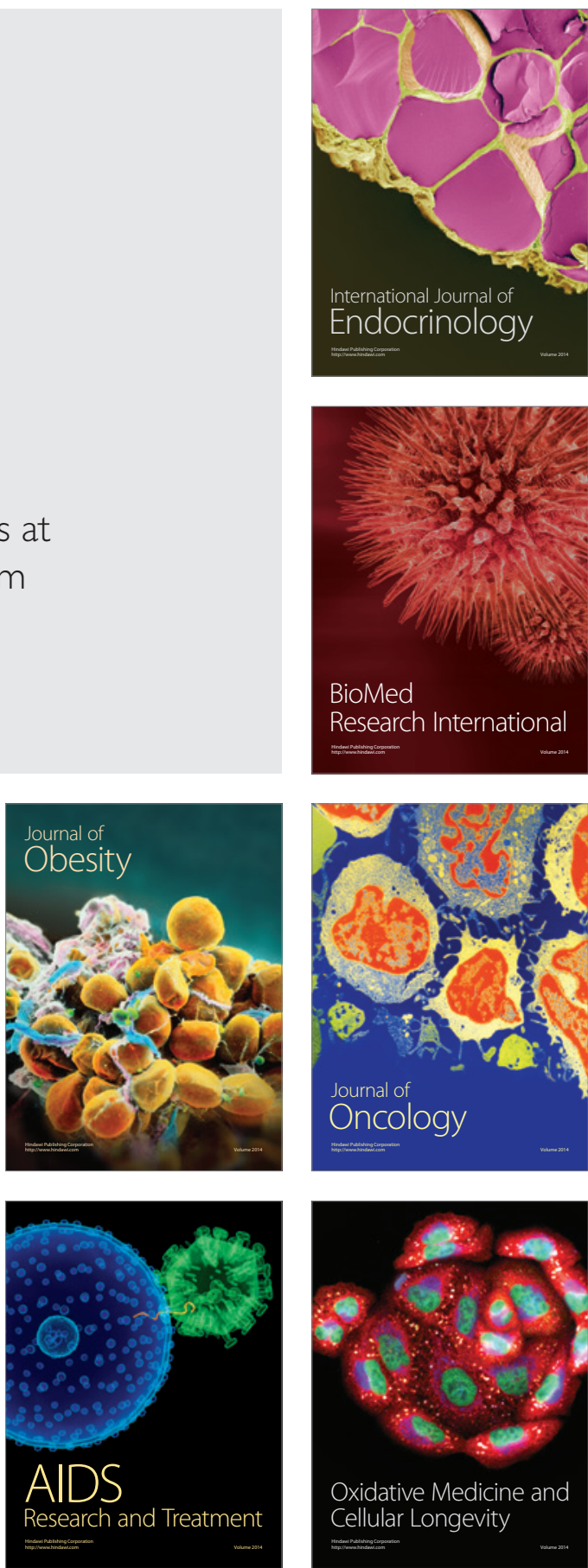